\title{
Le Tigre, le Louvre et l'échange de connaissances archéologiques visuelles entre la France et la Grande-Bretagne aux alentours de 1850
}

The Tigris, the Louvre and the exchange of visual archaeological knowledge between France and Britain around 1850

\section{Mirjam Brusius}

\section{CpenEdition}

Journals

Édition électronique

URL : http://journals.openedition.org/cel/404

DOI : $10.4000 / \mathrm{cel} .404$

ISSN : 2262-208X

Éditeur

École du Louvre

Édition imprimée

Date de publication : 1 octobre 2014

Référence électronique

Mirjam Brusius, « Le Tigre, le Louvre et l'échange de connaissances archéologiques visuelles entre la France et la Grande-Bretagne aux alentours de $1850 »$, Les Cahiers de l'École du Louvre [En ligne], 5 | 2014, mis en ligne le 01 octobre 2014, consulté le 20 avril 2019. URL : http:// journals.openedition.org/cel/404; DOI : 10.4000/cel.404

Les Cahiers de l'École du Louvre sont mis à disposition selon les termes de la licence Creative Commons Attribution - Pas d'Utilisation Commerciale - Pas de Modification 4.0 International. 


\section{Cahiers de l'École du Louvre}

recherches en histoire de l'art, histoire des civilisations archéologie, anthropologie et muséologie

\section{Numéro 5. Octobre 2014}

\section{Le Tigre, le Louvre et l'échange de connaissances archéologiques visuelles entre la France et la Grande-Bretagne aux alentours de 1850 Mirjam Brusius}

Article disponible en ligne à l'adresse :

http://www.ecoledulouvre.fr/cahiers-de-l-ecole-du-louvre/numero5octobre2014/Brusius.pdf

Pour citer cet article :

Mirjam Brusius, « Le Tigre, le Louvre et I'échange de connaissances archéologiques visuelles entre la France et la Grande-Bretagne aux alentours de 1850 », Cahiers de I'École du Louvre. Recherches en histoire de l'art, histoire des civilisations, archéologie, anthropologie et muséologie [en ligne] n 5, octobre 2014, p. 34 à 46.

\section{@creative \\ (․) $\ominus$}

(C) École du Louvre

Cet article est mis à disposition selon les termes de la Licence Creative Commons Attribution - Pas d'utilisation commerciale - Pas de modification 3.0 non transposé. 


\section{Cahiers de l'École du Louvre recherches en histoire de l'art, histoire des civilisations archéologie, anthropologie et muséologie \\ Numéro 5. octobre 2014}

\section{Sommaire}

\section{Introduction}

L'archéologie en construction : objets, images, dispositions

Nathan Schlanger

La collection de vases grecs du marquis de Northampton (1790-1851).

Entre archéologie et sciences de la nature

Marie-Amélie Bernard.

L'artisanat touristique du Sud-Ouest des États-Unis. L'exemple des objets collectés par Alphonse Pinart à Santa Fe, à la fin du XIX ${ }^{\mathrm{e}}$ siècle Éloïse Galliard

Regard sur les statuettes hindoues et bouddhiques en bronze d'Indonésie. Leur rôle pour la connaissance de la civilisation javanaise ancienne et ses liens avec l'Asie du Sud et du Sud-Est

Mathilde Mechling 25-33

Le Tigre, le Louvre et l'échange de connaissances archéologiques visuelles entre la France et la Grande-Bretagne aux alentours de 1850

Mirjam Brusius p. $34-46$

Le regard des photographes commerciaux. Quelques clichés du fonds égyptien de la Collection Fouad Debbas à l'étude.

Yasmine Chemali - Anne-Hélène Perrot

Émile Guimet et la morsure du canard égyptien.

Un curieux au musée de Boulaq

Thomas Lebée

L'exposition préhistorique de la Galerie de l'Histoire du travail en 1867. Organisation, réception et impacts 


\title{
Le Tigre, le Louvre et l'échange \\ de connaissances archéologiques visuelles \\ entre la France et la Grande-Bretagne \\ aux alentours de 1850
}

\section{Mirjam Brusius}

\begin{abstract}
«Le métal n’est plus fondu ou repoussé, des forces naturelles jusqu’alors ignorées le déposent par voie galvanoplastique. Au daguerréotype succède la talbotypie, qui le fait oublier, pour se faire oublier à son tour. "

Gottfried Semper, Science, industrie et art, 1851
\end{abstract}

Une trompette et un poisson enveloppés dans un tapis. Voici, nous dit la légende, tout ce qui est resté à Victor Place à la suite d'une perte majeure dans l'histoire de l'archéologie française. En 1853, et à nouveau en 1855, les fouilleurs Français avaient hissé de lourdes sculptures en pierre provenant de Dur-Šarruken (l'actuelle Khorsabad) sur des radeaux de bois afin de leur faire descendre le Tigre. Mais plusieurs de ces embarcations avaient coulé, entraînant la perte de deux grands taureaux ailés à figure humaine et de centaines de caisses. Le reste, quelques statues monumentales qui gardaient des portes et des stèles sur lesquelles se trouvent des bas-reliefs, est visible dans l'un des lieux les plus admirés du Louvre, la cour Khorsabad, qui contient des sculptures de la salle du trône du roi Sargon installées dans leur configuration d'origine. Quant aux objets perdus en 1855, ils n’ont jamais été retrouvés.

Dans le cadre de l'exploration de l'ancienne Mésopotamie, des fouilles archéologiques avaient déjà été entamées à Ninive et à Khorsabad dans les années 1840 par Paul Émile Botta, consul français à Mossoul et botaniste ${ }^{1}$. Outre celles des Français, des recherches étaient également entreprises par le Britannique Austen Henry Layard, explorateur aventureux, antiquaire et collectionneur, conduit par ses voyages à Bagdad dans les années 1840. Sans avoir initialement une vocation d'archéologue, Layard avait été fasciné par des monticules que Botta s'apprêtait à explorer près de Mossoul. Durant la campagne napoléonienne en Syrie et en Égypte entre 1798 et 1801, la France avait déjà entrepris des expéditions scientifiques, y compris la collecte d'antiquités égyptiennes - partiellement saisies par les Britanniques - qui avaient éveillé un grand intérêt pour l'Égypte et donné naissance à l'égyptologie.

Layard avait commencé ses premières fouilles à Nimrud - qu'il avait d'abord prise pour Ninive - en 1845, et en 1847 il commença à envoyer des artefacts à Londres ${ }^{2}$. L'assistait dans ses tâches Hormuzd Rassam, un habitant local qui reprit la responsabilité des fouilles au milieu des années $1850^{3}$. Les premières

1. Botta avait commencé à mettre au jour Koyoundjik, le monticule principal de Ninive, mais l'avait abandonné pour se rendre à Khorsabad, une vingtaine de kilomètres plus au nord après y avoir découvert le grand palais assyrien. Les Britanniques avaient décrit les résultats des fouilles françaises comme « de très peu d'importance », puisque Victor Place n'avait « ni expérience, ni connaissance des antiquités, ni même de passion pour l'entreprise dans laquelle il s'est engagé. », dans The British Museum Central Archives, mars 1852, OP, janvier à mai 1852. Cette déclaration laissait entendre que c'était exactement les qualités que les chercheurs britanniques possédaient et qui leur donnaient un sentiment de supériorité. Voir Thomas W. Davis, Shifting sands: the rise and fall of Biblical archaeology, New York, Oxford, Oxford University Press, 2004, p. 12

2. Pour Layard, voir Gordon Waterfield, Layard of Nineveh, Londres, John Murray, 1963 ; Nora Benjamin Kubie, Road to Nineveh: The adventures and excavations of Sir Austen Henry Layard, Garden City, Doubleday \& Co., 1964 ; Julian Reade, «Reflections on Layard's Archaeological Career », Frederick M. Fales, Bernard J. Hickey (ed.), Austen Henry Layard Tra L'Oriente E Venezia, International Symposium: Selected Papers, Roma, Rome, L'Erma di Bretschneider, 1987, pp. 47-53 ; ici p. 47 ; Mogens Larsen, The conquest of Assyria: excavations in an antique land, 1840-1860, Londres, Routledge, 1996, pp. 34-39.

3. Né dans une famille chrétienne à Mossoul et éduqué en Angleterre, Hormuzd Rassam est souvent décrit comme un intermédiaire ou passeur entre deux cultures, ce qui faisait de lui un personnage dont le rôle vaut la peine d'être exploré en détail ailleurs. Voir J. Reade, « Hormuzd Rassam and his Discoveries », Iraq 55, 1993, pp. 39-62; ici p. 59. 
cargaisons sont parvenues en Europe aux alentours de 1850. Les célèbres statues colossales n'étaient qu'une portion du produit des fouilles, en grande partie constitué de tablettes d'argile portant des inscriptions cunéiformes dans un alphabet qui n'avait pas encore été déchiffré à l'époque. À Londres et à Paris, les objets, autrefois fonctionnels, de l'une des cultures les plus anciennes qui soient connues ont été transformés en objets de musée appartenant aux plus importantes collections européennes d'antiquités. Layard et Botta recherchaient donc tous deux principalement des pièces pouvant être exposées respectivement au British Museum et au Louvre, surtout des statues et des bas-reliefs en pierre.

Ce critère manquait en fait de précision, dans la mesure où il était impossible d'évaluer par avance ce qu'il serait utile, approprié ou intéressant d'exposer dans un musée. Le passé de la région était généralement méconnu, de même que la place que pourraient occuper les objets dans les narrations muséologiques européennes. Contrairement à la France, où l'exposition et l'expansion des collections nationales étaient le moteur des recherches, en Grande-Bretagne il s'agissait aussi de retracer des récits bibliques. Pour ce pays, il était donc capital que les fouilles aient révélé d'importants lieux assyriens jusqu'alors uniquement connus par leur mention dans la Bible. Alors que l'intérêt porté à cette entreprise par les Britanniques était motivé par la foi et les croyances, en France, les missions scientifiques avaient un but non-religieux et nationaliste. Même si la recherche de lieux bibliques présentait un certain intérêt pour les Français, leur motivation relevait plutôt d'une poursuite croissante de la science positive, qui était venue à dominer l'archéologie : dans ce cadre, une importance particulière s'attachait à la mise au point de nouvelles technologies sous-tendues par l'idéologie républicaine - et en premier, nous le verrons, le procédé photographique

Les recherches menées dans l'ancienne Mésopotamie, à cette époque une province ottomane correspondant à l'Irak actuel, étaient avant tout motivées, pour les deux puissances européennes présentes dans la région, par un intérêt commercial et stratégique qui a fait de l'archéologie un « cas unique d'impérialisme informel $»^{5}$. Il n'est donc pas surprenant que ceux qui furent les premiers à creuser étaient aussi des hommes employés par la fonction publique. Les fouilles sont restées l'apanage d'un petit cercle de participants et d'un réseau d'acteurs travaillant dans les affaires étrangères qui ont façonné la perception par l'opinion publique des expéditions archéologiques. Ce point est important car il explique qu'il n'y a pas eu d'archéologue chevronné envoyé dans la région spécifiquement pour exhumer des vestiges archéologiques. L'archéologie fait ainsi partie d'une histoire bien plus vaste et complexe, dans laquelle les fouilles semblent parfois être plutôt des effets secondaires aléatoires.

Même si Layard et Botta ont apparemment continué à coopérer harmonieusement, l'historiographie insiste sur la concurrence entre les fouilles qui alla en augmentant au début des années 1850, lorsque les responsabilités ont changé et que les deux hommes n'étaient plus aux commandes ${ }^{6}$. Mais à bien des égards, les deux expéditions nationales ne peuvent clairement pas être considérées séparément l'une de l'autre, car les liens qui unissaient les deux pays étaient plus nombreux que ce qui les séparaient. Les valeurs culturelles, bien sûr, mais aussi des tentatives conjointes de déchiffrer l'écriture cunéiforme, par exemple, ou bien la conduite de fouilles. Dans ces deux pays, l’archéologie était une discipline « en

4. Voir John Tresch, The Romantic Machine: Utopian Science and Technology after Napoleon, Chicago, Londres, The University of Chicago Press, 2012, p. 287. Voir aussi Michael Osborne, «Science in the French Empire », Isis 96, 2005, pp. 80-87.

5. Margarita Diaz-Andreu, A world history of nineteenth-century archaeology: nationalism, colonialism, and the past, Oxford, Oxford University Press, 2007, p. 165. Pour l'archéologie et I'impérialisme, voir aussi M. Diaz-Andreu, Timothy Champion (ed.), Nationalism and Archaeology in Europe, Londres, UCL Press, 1996 ; Magnus Thorkell Bernhardsson, Reclaiming a plundered past: archaeology and nationalism in modern Iraq, 1808-1941, Austin, University of Texas Press, 2005. Pour les motivations des fouilles voir J. Reade, « Nineteenth-Century Nimrud: Motivation, Orientation, Conservation », J. E. Curtis, et al. (ed.), New Light on Nimrud, 11-13th March Proceedings of the Nimrud Conference, London, 2002, Londres, The British Museum, 2008, pp. $1-21$.

6. Voir Seton Lloyd, Foundations in the dust: a story of Mesopotamian exploration, New York, AMS Press, 1978, p. 113 ; J. Reade, « Les relations anglo-françaises en Assyrie » Élisabeth Fontan, Nicole Chevalier (dir.), De Khorsabad à Paris : La découverte des Assyriens, Paris, Réunion des Musées Nationaux, 1994, pp. 116-134; ici p. 121. 
devenir ", allant de pair avec l'évolution générale des sciences muséologiques et physiques. Autour de 1850, ni l'un ni l'autre des projets nationaux ne suivait de protocoles de fouilles techniques ou méthodologiques stricts. Enfin, le statut des découvertes en provenance de Mésopotamie n'était pas immédiatement évident à leur arrivée au musée, que ce soit en France ou en Grande-Bretagne. ${ }^{7}$

Un autre aspect, devenu une sérieuse épreuve partagée par les deux pays, concernait les difficultés de transport et de logistique. Devant les mêmes enjeux, il fallait pour les chercheurs pouvoir compter les uns sur les autres; les voyages étant rudes, il a été envisagé de partager les moyens de transport. Mais ces dispositions ont entraîné quelques confusions, quand des objets ont été expédiés à Paris alors qu’ils étaient censés partir pour Londres, et vice-versa ${ }^{8}$.

\section{La perte}

En 1855, par exemple, les Britanniques, manquant de moyens de transport, ont pu utiliser un radeau français pour l'acheminement de leurs pièces. Les Français, à leur tour, ont confié aux Britanniques quelques statues de Koyoundjik (Ninive). Mais de nombreuses sculptures ont disparu dans le Tigre?. Ceci a concerné principalement les découvertes des Français, même si les Britanniques ont également perdu des fragments. Ceux en provenance de Ninive, ont été soit égarés lors du transport, soit à leur arrivée en Grande-Bretagne ${ }^{10}$.

Un article paru dans la rubrique "Nos potins de la semaine » de l'hebdomadaire The Athenaeum nous donne un point de vue Britannique sur le naufrage : le bateau, apparemment surchargé et impossible à manœuvrer s'est donc échoué sur le rivage contre une haute berge un peu au-dessus de Korna, à la confluence du Tigre et de l'Euphrate. Il a ensuite sombré dans moins de dix mètres d'eau. L'Athenaeum rapporte que "les Arabes des alentours, voyant le naufrage, sont aussitôt descendus partager le butin, et après avoir pillé le bateau qui coulait, ont attaqué les radeaux qui l'accompagnaient, arrachant les peaux de bêtes gonflées dans l'espoir de trouver des trésors - entrainnant ainsi les caisses et les marbres lourds qui ont coulé au fond de la rivière ». Seuls un ballot et six petites caisses ont atteint Bassora intacts. L'espoir de récupérer une partie des objets coulés s'est avéré vain. Ce rapport dramatique se termine cependant par un adoucissement remarquable : "Malgré la douleur ressentie par tous les amoureux de la science à l'évocation de cet accident, c'est une consolation de savoir que M. Place a pris des photographies des marbres de Khorsabad, tandis que des dessins de ceux de Koyoundjik ont été esquissés par M. Boutcher, l'artiste du British Museum ${ }^{11}$ ».

Le propos de notre article est donc d'examiner l'utilisation de supports visuels concernant ces objets perdus. En effet, les deux expéditions étaient accompagnées d'artistes chargés de rendre compte des sites des fouilles et des découvertes. La photographie et le dessin étaient utilisés, mais leur usage n'était pas valorisé de la même façon dans les deux pays. L'une des principales raisons pour lesquelles il semblait important d'employer des artistes était précisément la crainte constante de perdre les objets eux-mêmes. Comme nous l'avons vu, cette crainte n'était pas sans fondement. Deux éléments de cette histoire sont à remarquer : d'abord, si les fragments perdus par la suite ont été enregistrés dans les deux pays, il est arrivé, dans certains cas, que ces images aient été faites par le pays " concurrent ", et non pas par celui qui avait subi la perte. Ensuite, si des nouvelles techniques visuelles comme la photographie commençaient à être utilisées, elles se répartissaient,

7. Voir Mirjam Brusius, « Misfit Objects: Excavations in Mesopotamia and biblical imagination in mid-19th century Britain », Journal of Literature and Science 5, 2012, n 1, pp. 38-52.

8. Voir lettre de Loftus à Ellis, Morland Cottage, 16 octobre 1855, The British Museum Central Archives, OP, juillet 1855-mars 1856.

9. Voir J. Reade, « More drawings of Ashurbanipal sculptures », Iraq 26, 1964, pp. 1-13 ; ici p. 2. Pour la question du transport, voir N. Chevalier, « La folie franque », É. Fontan, N. Chevalier (dir.), op. cit. note 6, pp. 214-225.

10. Voir J. Reade, art. cit. note 6, p. 134. Voir aussi J. Reade, « New lives for old stones », Iraq 72, 2010, pp. 163-174 ; ici pp. 165-167. Pour une liste de photographies des fragments perdus, voir J. Reade, op. cit. note 9, Appendice.

11. The Athenaeum, n 1448, 28 juillet 1855, p. 877. Voir aussi J. Reade, art. cit. note 6, p. 131. 
à l'instar de certain des vestiges, des deux côtés de la Manche. Notre histoire est donc faite d'échanges et d'entrecroisements.

Bien que les vestiges en eux-mêmes aient servi de "données ", de preuves matérielles (par exemple de la véracité de la Bible), leurs images étaient plutôt considérées comme des "données de second ordre ", un substitut adéquat au cas où un élément viendrait à disparaitre, et aussi un moyen de donner un sens aux découvertes à travers leur représentation visuelle. L'un des moyens les plus répandus à l'époque d'enregistrer, sur papier, le résultat des fouilles était le dessin. Ce dispositif d'enregistrement individuel aidait à comprendre, interpréter et contextualiser les résultats des fouilles. Britanniques et Français employaient depuis des années des dessinateurs. Eugène Flandin de l'Académie des Inscriptions et Belles-Lettres, le principal dessinateur au service des Français ${ }^{12}$, effectuait deux types de travaux : d'une part, les vues générales des bas-reliefs in situ et d'autre part, des fragments de sculptures spécifiques. La qualité de ses images était tout à fait convaincante ${ }^{13}$, au point que Layard s'avouait " plein de honte quand je compare mes dessins publics à ceux des Français ${ }^{14}$ ".

Mais la France et la Grande-Bretagne, à la pointe de l'archéologie en Mésopotamie, étaient également les principaux acteurs de l'invention de la photographie. Pour tous deux, cependant, ce nouvel outil est entré dans le domaine archéologique à un moment où n'étaient pas encore clairement définies les méthodes entourant cette technique de visualisation et les fonctions qu'elle pouvait prendre pour la formation de la discipline. Il n'était pas évident que la photographie ait un rôle à jouer, et quel rôle, pour l'archéologie. En France, c'est grâce aux travaux de l'artiste Louis-Jacques-Mandé Daguerre que le public a fait la connaissance de cette nouvelle technique, par l'intermédiaire du daguerréotype, un processus qui, grâce à une camera obscura, permettait de restituer de minuscules détails et produisait des images uniques, mais qui n'était pas reproductibles. En 1839, cependant, François-Dominique Arago, le secrétaire perpétuel de l'Académie des Sciences, pouvait déjà prédire que le daguerréotype français deviendrait un objet d'usage courant et même indispensable aux voyageurs, archéologues et naturalistes :

"Pour copier les millions de hiéroglyphes qui couvrent, même à l'extérieur, les grands monuments de Thèbes, de Memphis, de Karnak, etc., il faudrait des vingtaines d'années et des légions de dessinateurs. Avec le daguerréotype, un seul homme pourrait mener à bonne fin cet immense travail. Munissez l'institut d'Égypte de deux ou trois appareils de M. Daguerre, et sur plusieurs des grandes planches de l'ouvrage célèbre, fruit de notre immortelle expédition, de vastes étendues de hiéroglyphes réels iront remplacer des hiéroglyphes fictifs ou de pure convention ; et les dessins surpasseront partout en fidélité, en couleur locale, les œuvres des plus habiles peintres ; et les images photographiques, étant soumises dans leur formation aux règles de la géométrie, permettront, à l'aide d'un petit nombre de données, de remonter aux dimensions exactes des parties les plus élevées, les plus inaccessibles des édifices ${ }^{15} \%$.

Même si les observateurs externes considéraient souvent cette technique comme utile, "les utilisateurs experts " - dans la mesure où ils existaient dans le domaine de l'archéologie - adoptaient souvent un point de vue différent. Botta, par exemple, trouvait la photographie inadaptée à une utilisation sur les sites archéologiques en raison des difficultés d'accès de l'équipement sur le palais en cours de fouille : "le daguerréotype lui sera bien inutile, rien n'est dessinable de cette manière parce que les passages sont si étroits que nulle part on ne peut le

12. Pour Flandin, voir Pauline Albenda, « Les dessins de Flandin », É. Fontan, N. Chevalier (dir.), op. cit. note 6, p. 184-195. Françoise Demange, «Eugène Flandin, un peintre archéologue », É. Fontan, N. Chevalier (dir.), op. cit. note 6, p. 86-93.

13. Voir P. Albenda, art. cit. note 12, p. 184-195.

14. J. Reade, art. cit. note 6, p. 124.

15. François-Dominique Arago, Historique et description des procédés du Daguerréotype et du Diorama par Daguerre, Paris, A. Giroux et Cie, 1839. Voir aussi Lorraine Daston, Peter Galison, Objectivity, New York, Cambridge (Mass.), Zone Books, 2007, p. 34. 
mettre au point de vue, c'est comme si on voulait daguerréotyper dans des égouts dont les murailles seraient sculptées ${ }^{16} "$.

Un scepticisme envers la photographie régnait aussi en Grande Bretagne. En 1839, l'érudit anglais William Henry Fox Talbot était apparu devant des sociétés savantes à Londres pour annoncer qu'il avait lui-même travaillé, des années auparavant, sur un procédé comparable, dont les images s'accompagnaient de négatifs reproductibles. Sa technique, le calotype (d'abord appelée Talbotype), s'était cependant avérée moins précise pour restituer des détails avec contraste. Talbot, un antiquaire très lié au British Museum, était comme Arago partisan de l'utilisation de la photographie dans le domaine archéologique. Cependant, il n’a pas été jugé essentiel d'utiliser ce nouveau support pour les fouilles. Pendant une dizaine d'années environ, les archéologues avaient eu connaissance de ces techniques, sans pour autant leur porter attention ${ }^{17}$.

Les Britanniques avaient travaillé avec plusieurs artistes, dont beaucoup ne restaient que très peu de temps sur le terrain avant de repartir. Il s'agissait uniquement de dessinateurs; la photographie était à peine mentionnée dans les rapports sur les fouilles. C'est seulement en 1854, quand un dessinateur a quitté le site des excavations britanniques en raison de problèmes de santé et qu'un nouvel artiste a été sollicité, il fût demandé que celui-ci soit un dessinateur ou " un photographiste " - une désignation qui indique bien une incertitude terminologique, le mot " photographe " étant déjà utilisés à l'époque. Le British Museum nomma William Boutcher "son dessinateur ", tout en incluant la photographie dans son mandat. La façon dont celle-ci est mentionnée mérite attention : on demande à Boutcher de «faire des dessins ou des photographies de tous les objets [destinés à être] relevés ${ }^{18}$ ".

La conjonction « ou » montre bien que le choix de l'une ou l'autre technique n'était pas arrêté. D'autres indications suggèrent que la nature du médium utilisée pour documenter les fouilles archéologiques britanniques n'importait guère, que ce soit sur le terrain ou dans le musée. Lorsque pour la première fois, le British Museum a demandé explicitement des photographies, elles ont été considérées au même titre que les dessins dans leur rôle de substituts potentiels aux objets originaux. Ceci confirme bien que la photographie n'était pas toujours considérée comme un médium exceptionnel.

Cela ne signifie pourtant pas que les archives du British Museum concernant ces premières fouilles ne contiennent pas de photographies. Les rares exemplaires qui ont survécu sont décolorés au point d'être presque totalement indiscernables, et seules quelques lignes vagues rappellent les panneaux qu'elles représentaient autrefois. Même extrêmement pâlies aujourd'hui, ces photographies constituent des documents importants. Deux gravures (fig. 1) ont été faites d'après les deux photographies sépia fanées mentionnées ci-dessus. On a longtemps cru que ces clichés avaient été pris par Boutcher en 1854, parce que les gravures effectuées d'après elles portent sa signature en bas à gauche.

16. Lettre de Botta à Mohl, 5 octobre 1843. Cité dans N. Chevalier, Bertrand Lavédrine, «Débuts de la photographie et fouilles en Assyrie : les calotypes de Gabriel Tranchand », E. Fontan, N. Chevalier (dir.), op. cit. note 6, pp. 196-213; ici p. 196.

17. Voir M. Brusius, « Inscriptions in a double sense: An early scientific photograph of script », Nuncius, Journal of the History of Science 24, 2009, n², pp. 367-392; M. Brusius, « From Photographic Science to Scientific Photography: Photographic experiments at the British Museum around $1850 »$, M. Brusius, Katrina Dean, Chitra Ramalingam (ed.), William Henry Fox Talbot: Beyond Photography, New Haven, Londres, Yale University Press, 2013, pp. 219-244.

18. Nous soulignons. Extrait du mandat de M. Dickinson, The British Museum Central Archives, OLP vol. 51, juin-décembre 1854. Voir aussi le compte rendu, 8 avril 1854, British Museum, C 6. Le département du Moyen-Orient du British Museum possède un volume contenant deux photographies originales (Or. Dr. VII, The Royal Asiatic Society Portfolio, pl. XX et XXVI). Ces photographies sont discutées et commentées (recopiées d'après l'original) avec des dessins de Boutcher dans R. D. Barnett, Sculptures from the north palace of Ashurbanipal at Nineveh (668627 BC), Londres, The British Museum Publications, 1976. Pour Boutcher, voir aussi J. Reade, art. cit. note 9 ; Id., art. cit. note 5 ; Id., art. cit. note 10. M. Brusius, Preserving the Forgotten: William Henry Fox Talbot, Photography, and the Antique, thèse de doctorat, University of Cambridge, 2011. Chapitre III. 
Elles représentent deux stèles cassées sur lesquelles figure une procession assyrienne. La plus grande partie du panneau inférieur a été perdue pendant le transport de l'objet, ce qui fait de ces images, au moins des gravures, un témoignage capital pour le British Museum ${ }^{19}$. La gravure supérieure représente les panneaux sculptés à côté de l'ombre portée d'un panneau attenant, le tout devant un mur en briques de terre sombre. Comme nous le savons grâce à une reproduction faite avant que la photo ne se fane, ce fond superflu apparaît sur la photographie d'origine comme une masse étonnamment distrayante, ce qui ne rend pas nécessairement cette image plus instructive.

Sur la gravure inférieure, des parties de la section supérieure du panneau ont été ajoutées. En général, les gravures comportent certains rajouts tandis que d'autres détails sont omis. Nous ne savons pas si la signature de Boutcher porte sur la photographie ou sur la gravure, mais cette précision ne revêtait probablement pas une grande importance, dans la mesure où la photographie n'était pas tenue pour plus crédible que le dessin. En réalité, la question de la crédibilité de la photographie ne semblait pas être d'actualité en Grande-Bretagne : la photographie n'était pas un dispositif d'enregistrement auto-suffisant, puisque l'apparence originale des images qu'elle produisait pouvait être modifiée par la gravure. Nous pouvons conclure qu'autour de 1850, la différence entre ces deux techniques, la photographie et le dessin, n'était pas immédiatement apparente à leurs utilisateurs, du moins en Grande-Bretagne. 
Un autre fait intéressant est que nous avons de bonnes raisons de douter que Boutcher soit réellement l'auteur de ces photographies. Boutcher, qui était censé emporter avec lui un appareil photographique, avait, à un certain moment, demandé un nouvel instrument en provenance de Paris qu'il espérait voir mieux fonctionner dans le climat imprévisible de la région. Cependant, cet appareil ne lui est pas parvenu pendant son séjour ${ }^{20}$. Après son retour à Londres, Boutcher s'est excusé dans une lettre au British Museum du fait que l'appareil ne soit arrivé qu'après son départ de Ninive. Mais qui a donc pris ces photographies ? Étant donné que la France et la Grande-Bretagne dépendaient l'une de l'autre à bien des égards, ce qui suit ne sera peut-être pas une surprise.

\section{Le daguerréotype sur papier}

Alors que l'histoire de la photographie britannique en Mésopotamie s'était révélée plutôt infructueuse, les Français avaient une relation moins compliquée avec cette technique. Les conservateurs du British Museum n'avaient pas porté grand intérêt au procédé de Talbot, le calotype. Et tandis qu'Arago est resté partisan du daguerréotype, c'est en France, plutôt ironiquement, que Talbot a trouvé un vrai défenseur de son procédé : l'homme de sciences Jean-Baptiste Biot (17741862) l'a défendu plus que quiconque outre-Manche ${ }^{21}$. Biot a fait état de la correspondance de Talbot à l'Académie des sciences, et a montré son travail à certains de ses collègues à Paris. Le procédé de reproduction sur papier lui semblait décisif, d'autant plus que les copies effectuées par Talbot étaient aussi lisibles que les textes d'origine ${ }^{22}$. Biot a été particulièrement intrigué par l'idée de Talbot d'utiliser la photographie comme une forme d'écriture invisible lorsqu'elle est exposée à la lumière du soleil23. Mais, surtout, les deux hommes partageaient la conviction que la photographie pouvait servir les découvertes scientifiques. Par conséquent, en France, l'utilisation du calotype lors d'expéditions archéologiques est devenue plus courante que celle du daguerréotype, car bien que manquant de netteté cette technique était considérée comme plus pratique, en raison de la légèreté des négatifs papier et de la possibilité de leur reproduction.

Il a été suggéré par Julian Reade que les photographies de Ninive, sur lesquelles Boutcher s'est appuyé pour ses gravures, ont, en fait, été prises en $1854^{24}$ par les Français, dont la fouille à Khorsabad était distante d'une cinquantaine de kilomètres seulement de Ninive. Il est probable que Gabriel Tranchand, le photographe et ingénieur qui accompagnait l'archéologue Victor Place sur les fouilles, ait fait don de ces photos au British Museum. De ce fait, les documents les plus importants conservés en Grande-Bretagne sur ces fragments partiellement perdus pourraient fort bien provenir des Français.

Nicole Chevalier et Bertrand Lavédrine ont montré pour leur part le rôle important qu’a joué la photographie dans les expéditions de Victor Place ${ }^{25}$. Contrairement à Paul-Émile Botta son prédécesseur, Place considérait la photographie comme un médium approprié à l'archéologie. Ayant entendu parler " des vues qui offrent avant tout aux savants la garantie d'une exactitude mathématique ", il estimait la photographie "indispensable dans la découverte archéologique $»^{26}$.

20. Lettre de Loftus à Rawlinson, 28 septembre 1854, The British Museum Central Archives, OP, juin-décembre 1854.

21. Voir John Tresch, « The Daguerreotype's first frame: Francois Arago's moral economy of instruments », Studies in History and Philosophy of Science , 2007, pp. 445-476.

22. Jean-Baptiste Biot, « Notes sur des dessins photogéniques de M. Talbot », CRAS 10, 1840. Cité dans Theresa Levitt, The shadow of enlightenment: optical and political transparency in France 1789-1848, Oxford, Oxford University Press, 2009, p. 150.

23. Voir T. Levitt, op. cit. note 22, p. 152.

24. J. Reade, art. cit. note 10, p. 169. Gadd présume qu'aucune photographie n'a été prise par les Britanniques. C. J. Gadd, The Stones of Assyria: The surviving remains of Assyrian sculpture, their recovery, and their original positions, Londres, Chatto \& Windus, 1936, p. 111.

25. N. Chevalier, B. Lavédrine, art. cit. note 16, p. 196-213.

26. Voir N. Chevalier, B. Lavédrine, art. cit. note 16, p. 196. Archives nationales, F 21, 546, Place au ministre de l'intérieur, 9 septembre 1851. Voir aussi Marc Pillet, Un pionnier de l'assyriologie Victor Place, consul de France à Mossoul, explorateur du palais de Sargon II (722-705 av. J.-C.) à Khorsabad (1852-1855) avec les photographies originales de M. Tranchand, Cahiers de la Société Asiatique XVI, Paris, Imprimerie Nationale, 1957. Pour Place, voir aussi N. Chevalier, «Victor Place : consulat et archéologie », É. Fontan, N. Chevalier (dir.), op. cit. note 6, pp. 94101 
En 1853, Victor Place a pris connaissance du procédé au collodion, qui, du fait de sa reproductibilité, était le seul procédé qui permettait de restituer les sites assyriens et les rendre accessibles ${ }^{27}$.

Pour Victor Place, les photos étaient simplement un autre genre de dessin, mais d'une extraordinaire fidélité : "Mon but étant principalement de fournir à la science des données positives, en écartant soigneusement tout ce qui ne serait qu'hypothétique, il était essentiel que les plans et les descriptions fussent accompagnés de dessins qui comportassent non seulement une grande certitude mais la preuve même de cette certitude, et rien n'atteignait mieux ce résultat que des vues daguerriennes ${ }^{28} »$. Tranchand a fini par accompagner Place en guise d'alter ego photographe, faisant partie de l'équipe tout en observant de loin. Pour ce qui est de l'équipement photographique, Tranchand avait rencontré des difficultés similaires à celles déjà affrontées par les photographes britanniques ${ }^{29}$ : la logistique était difficile à organiser, les artistes souffraient constamment de maladies, et le projet dans son ensemble était chaotique et par moment imprévisible ${ }^{30}$. C'est pour des raisons toutes opposées que le choix a été fait d'utiliser le calotype, pour rendre compte de l'avancée et de la réussite des fouilles, comme le suggère cette image par Tranchand (fig. 2).

Les incertitudes entourant la photographie se sont également manifestées dans la terminologie utilisée en France. Le fait que les Français aient développé leur propre technique, mais qu'ils aient néanmoins utilisé le procédé sur papier des Britanniques, était une source de confusion mentionnée dans des revues contemporaines : "Nous n'avons pas choisi le mot photographie [...] il signifie en France, en Allemagne et en Belgique, Daguerréotype sur papier ${ }^{31}$ ».

Pour Place, Tranchand était bien plus qu'un photographe. Sans rien omettre ni ajouter, la photographie permettait de contrôler les fouilles, et de garantir l'exactitude sur laquelle Place insistait. Une telle foi en la véracité de la plaque photographique n’avait pas encore été exprimée par les Britanniques, du moins à un tel degré : comme le dit Place « la vue a peu de relief, mais j’ai préféré cela à un dessin, afin que l'Académie fût parfaitement certaine que rien n'avait été ajouté ni retranché. [...] Je tiens absolument à ce que toutes les vues que vous recevrez aient ce degré d'exactitude que la photographie seule peut donner ${ }^{32}$ ».

Convaincu de leur utilité, Place inclus certaines de ces photographies en tant que lithographies dans son livre, Ninive et l'Assyrie (1867-1870), tout comme Layard l'avait fait avec les dessins de ses artistes. L'ouvrage de Place est illustré par des gravures réalisées d'après des photographies, mais certaines d'entre elles - comme les gravures de Boutcher - comportent des divergences notables. Pourtant, Place prétendait montrer une image exacte de l'état dans lequel un objet a été trouvé33. Il eut recours à des êtres humains, en l'occurence des ouvriers arabes locaux, pour indiquer la taille des découvertes (fig. 2). Il semble donc que pour Place, l'« exactitude » concernait la précision mathématique de l'échelle utilisée et le fait

27. Voir M. Pillet, op. cit. note 26, p. 106. Ernest Lacan, « La mission de M. Place». La Lumière. Revue de la photographie, $\mathrm{n}^{\circ} 16$, novembre 1851 , p. 3.

28. Archives nationales, F 21,547, Rapport PLACE № 41, 18 décembre 1855. Aussi cité dans N. Chevalier, B. Lavédrine, art. cit. note 16, p. 196.

29. Certaines photographies ont pu être prises par Place lui-même ou par Félix Thomas, même si Tranchand est probablement celui qui les a développées. Voir M. Pillet, art. cit. note 26, pp. 105, 111. N. Chevalier, B. Lavédrine, art. cit. note 16; Tranchand a été peu étudié, mais il est aussi mentionné dans Nissan N. Perez, Focus East: early photography in the Near East (1839-1885), New York, Abrams, 1988, p. 227. M. Larsen, op. cit. note 2, pp. 307-309, p. 314, p. 351. La plupart des originaux des photographies de Tranchand ont été perdus. Certains d'entre eux, dont la liste figure dans l'ouvrage de Pillet, mais surtout des copies modernes de ces photographies sont conservées dans les Archives nationales (F. 546 et 547) et au Collège de France. Ces deux institutions possèdent également des documents manuscrits (lettres, journaux, rapports) concernant les fouilles.

30. N. Chevalier donne des descriptions ahurissantes des difficultés rencontrées par les archéologues français, Place en particulier. Voir N. Chevalier, art. cit. note 9.

31. Anonyme, La Lumière. Revue de la photographie, $\mathrm{n}^{\circ} 20$, avril 1851. Voir aussi Geoffrey Batchen, «The Naming of Photography: "a Mass of Metaphor" », History of Photography 17, $1993, n^{\circ} 1$, pp. 22-32.

32. Archives nationales, F 21, 546 1851-1852, Rapport № 7. Voir aussi N. Chevalier, B. Lavédrine, art. cit. note 16, p. 208.

33. Frederick Bohrer, «Edges of Art: Photographic Albums, Archaeology, and Representation », Stephen Bann (ed.), Art and the Early Photographic Album, New Haven, Londres, Yale University Press, 2011, pp. 222-235 ; ici pp. 225-226. L. Daston, P. Galison, op. cit. note 15, pp. $125-138$. 
que les fouilles étaient documentées au moyen d'un " appareil " photographique, plutôt que la précision et la netteté du cliché en lui-même, ou même le statut de la photographie en tant que témoignage documentaire. Effectivement, les originaux passés des gravures mentionnées ci-dessus représentent un cas où la photographie n’a pas réussi à se focaliser (dans les deux sens du terme) sur ce qui représentait de l'intérêt. La précision accrue de la photographie se trouvait avoir un inconvénient évident : elle ne permettait pas de distinguer l'essentiel du superflu, ce superflu étant parfois un surplus inutile de détails visuels. En effet, la précision pouvait rendre des dégradations physiques ou des altérations aléatoires bien visibles, parfois même plus qu'elles ne l'étaient en réalité. De ce fait, la perfection de la mise en scène allait parfois à l'encontre de l'" exactitude mathématique " à laquelle Place aspirait. Comme pour les dessins de Layard, il transparaît des photographies publiées par Place que le travail de terrain devait parler au public en métropole. Ces images étaient tenues de montrer que les équipes savaient exactement ce qu'elles faisaient. En tant que protocoles visuels d'une mission se voulant professionnelle, ces photographies avaient pour rôle d'attester du bon déroulement des fouilles.

Certaines de ces photographies ont donc été prises en série, pour montrer les différentes étapes de la fouille : l'entrée d'un palais à peine mise au jour, une ouverture en partie visible, des photographies mises en scène où l'équipe française et les ouvriers locaux posent devant la porte du palais, et enfin Place lui-même posant sur un amas de vestiges exhumés, symbole de l'impérialisme, comme la destination finale de l'entreprise. Si le réalisme n'était pas considéré comme intrinsèquement supérieur, les photographies de Tranchand restent remarquablement variées, allant de ladite série à des paysages, des gros plans du site ou des clichés de petit mobilier.

Cependant, des difficultés financières comparables à celles qu'avait connues le British Museum, menèrent rapidement à l'arrêt temporaire des activités photographiques au milieu des années $1850^{34}$. On peut soutenir que les photographies de Tranchand ont été les premières expériences visuelles, sur le terrain, à avoir testé les usages potentiels de la photographie archéologique. Il est 
remarquable de constater à quel point certaines de ses photographies ne diffèrent pas des dessins idéalisés et orientalisés de Layard. Et curieusement, ce ne sont pas les photographies de Tranchand, mais plutôt les dessins succincts de Place qui ont servi d'inspiration aux reconstructions futures de l'architecture assyrienne ${ }^{35}$.

Cela montre combien l'importance des dessins n'était aucunement diminuée par l'émergence de la photographie. Ainsi, William Boutcher, qui n'avait sans doute jamais vu un appareil photographique, avait dessiné plusieurs stèles dans leur état complet, alors que le Louvre et le British Museum n'en possèdent que des fragments. Il arrivait que des bas-reliefs soient arrachés, l'un de leurs morceaux expédié à Londres et l'autre à Paris. Dans de tels cas, un dessin ou une photographie, exécuté par les Français ou par les Britanniques, demeurait le seul document montrant les vestiges dans leur intégralité.

L'objet référencé AO 19903 au Louvre, par exemple, est un fragment du 124886-7 (fig. 3) du British Museum. Boutcher a dessiné la scène de chasse des deux morceaux lorsqu'ils étaient encore ensemble à Ninive (Koyoundjik). Depuis son dessin, la pièce du Louvre a perdu plusieurs fragments (fig. 4) qui représentent les panneaux muraux entiers d'une chasse aux lions. Tout comme Tranchand aurait pu donner aux Britanniques des photos de fragments entretemps égarés, Boutcher a offert au Louvre des dessins des fragments de bas-relief perdus dans le Tigre :

"Votre Majesté Impériale aura sans doute entendu parler de la perte regrettée dans le Tigre de la magnifique collection de sculptures et d'autres articles expédiés depuis l'Assyrie par le consul de France. Certaines des sculptures ont été photographiées par l'artiste français. Du reste (environ 70 caisses) il ne subsiste aucune trace, sauf les dessins que j'ai produits en Assyrie pendant que j'étais attaché comme artiste au British Museum et à l'Assyrian Excavation Society. Dès que j'ai entendu parler de cette perte, j’ai pensé que votre Majesté Impériale souhaiterait probablement posséder des copies des sculptures. Si c’est le cas, votre Majesté Impériale peut requérir mes services ${ }^{36} »$. 
William Boutcher Ninive, Palais nord, plece S1, C. dessin (crayon sur papier gris) début des années 1850 H. 0,528 . L $0,366 \mathrm{~m}$. Londres, The British Museum, Londres, The Brish Museum Museum Number 2007, 6024.454 , Or. Dr.V.4

Ninive Palais nord, pièce S1, D. dessin (crayon sur papier gris) début des années 1850 H. 0,533 ; L. $0,366 \mathrm{~m}$. Londres, The British Museum
Museum Number 2007,6024.470, Or. Dr.V.20 (Original Drawings vol. V, drawing 20) (c) Trustees of the British museum
Un autre exemple d'images détenues par les Britanniques est une gravure (fig. 5) - sans doute d'après un dessin de Boutcher réalisé sur le site des fouilles - publié dans The Illustrated London News ${ }^{37}$ et intitule Part of the Garden. On y voit une stèle complète, alors que les Français n'en possédaient qu'une photographie sur laquelle manque un important fragment. Nous savons cela grâce à une gravure montrant la dalle d'où le fragment est absent, gravure qui serait faite d'après une photographie probablement prise à Ninive en 1854-1855 par le français Tranchand (fig. 6) ${ }^{38}$. Mais il n'est pas précisé que ces morceaux ont sombré dans le Tigre. Reade suggère que : «Peut-être pour une raison quelconque, un mauvais état, par exemple ils n'ont pas été emballés ou envoyés ». Il se peut cependant qu'ils aient été perdus en cours de route, voire égarés ou même jetés par inadvertance après leur arrivée à Londres, n'étant pas très ouvragés : des cas semblables sont connus au XIX siècle, où les autorités ou le personnel du British Museum ont reçu en provenance de monuments anciens de simples morceaux de pierre dont ils ne savaient pas vraiment que faire ${ }^{39}$. Reade estime qu'il n'est pas inconcevable que les fragments manquants ne soient pas dans le Tigre, mais en réalité quelque part sur le site du British Museum. Qu'il s'agisse de dessins ou de photographies est, dans ce cas, de moindre importance par rapport au fait que les photographies de Tranchand ou les dessins de Boutcher représentaient des substituts visuels à ce qui a constitué une perte majeure dans l'histoire des fouilles en Mésopotamie au XIX ${ }^{\mathrm{e}}$ siècle. 


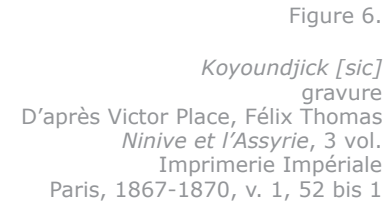

Le sort des antiquités rassemblées par les Français et perdues dans le Tigre était encore dans tous les esprits quand, dans les années 1870, Talbot tenta à nouveau d'encourager l'usage de la photographie pour les fouilles du British Museum. Puisque les stèles étaient loin, la photographie représentait pour lui des atouts tels que la préservation, la vitesse et la victoire sur l'éloignement. Mais c'est le traumatisme de la perte qui lui fournissait son argument principal. L'emploi d'un photographe permettrait de " parer au risque de voir les antiquités perdues dans un naufrage, en transit, comme ce fut le cas pour la collection française dont le transport a coulé dans le Tigre à cause de la négligence des bateliers arabes. Qui plus est, les inscriptions ou du moins leur premier versement pourraient ainsi rejoindre le musée beaucoup plus tôt $t^{40}$ ". Les démarches de Talbot montrent d'une part, l'importance des illustrations en tant que substituts, et d'autre part, que l'utilisation de la photographie sur le terrain archéologique n'était toujours pas une évidence dans les années 1870.

L'histoire racontée ici montre avant tout que la France et la Grande-Bretagne avaient besoin des techniques d'enregistrement visuel pour donner un sens à leurs découvertes. Mais les tournants décisifs et les hasards remarquables de cet épisode ne concernent pas seulement les échanges (en partie accidentels) de vestiges et d'images, mais aussi le partage de connaissances technologiques, dont la photographie. Si Tranchand avait, en effet, fait don au British Museum des deux photographies prises à Ninive en 1854 comme substitut aux fragments perdus par les Britanniques, cela indiquerait que l'invention de Talbot, négligée dans son pays d'origine, était passée de Grande-Bretagne en France avant d'y retourner, en transitant par la Mésopotamie. Considérant la " calotypomanie ${ }^{41}$ " française, surtout lorsqu'il s'agissait de documenter des monuments anciens à la suite de Tranchand, on pourrait aussi suggérer que ce détour du calotype par la France était indispensable à son avenir en Grande-Bretagne, même si celui-ci fut bref. Dans ce contexte, attribuer les rares spécimens photographiques du British Museum à leurs auteurs est moins important que constater à quel point la technique photographique n'était pas envisagée à l'époque comme un médium définitif, une " fin en soi ». Au contraire, la photographie était considérée comme une étape intermédiaire, destinée à être dépassée et remplacée par d'autres procédés plus établis, tels le dessin ou la gravure.

L'histoire de la photographie sur le terrain de l'archéologie contredit l'hypothèse répandue selon laquelle les nouveaux médias suivent un parcours téléologique et se voient immédiatement et couramment utilisées dès leur apparition. Le manque d'eau propre, les appareils photographiques perdus, les images pillées, les artistes épuisés et malades, tout cela constituaient des obstacles pratiques à la photographie. Alors que transparaît, dans les lettres envoyées depuis le British Museum, un

40. Lettre de Talbot à Birch, 22 octobre 1876, Talbot Correspondence, Doc. № 1239,

http://foxtalbot.dmu.ac.uk/letters/letters.html.

41. Pour le rôle du calotype en France, voir Abigail Solomon-Godeau, « Calotypomania: The Gourmet Guide to Nineteenth Century Photography », Afterimage 11, 1983, n Summer, pp. 7-12. Pour la Grande-Bretagne, voir Roger Taylor, Impressed by light: British photographs from paper negatives, 1840-1860, New Haven, Londres, Yale University Press, 2007. 
besoin urgent d'envoyer des artistes sur le terrain, l'indifférence exprimée envers la photographie en tant que technique privilégiée montre que son usage n'avait absolument rien d'urgent. Loin d'être une révélation qui se suffisait à elle-même, il s'agissait là d'un outil parmi d'autres disponibles dans le domaine archéologique de l'époque. En tant que nouveau médium la photographie s'est jointe au chaos qui régnait sur le terrain comme un intervenant supplémentaire, apportant avec elle son lot d'incertitudes, plutôt que comme un outil abouti, prêt à résoudre une difficulté. Il semble que la photographie n'était pas tant la solution qu'une partie du problème : en fait, la connaissance des objets, des traces et des matériaux dépend bien plus d'intérêts et de préoccupations humaines, que de systèmes techniques pris isolément. Ce sont des évaluations spécifiques et souvent complexes de la combinaison appropriée de techniques photographiques avec les éléments à enregistrer qui orientent et déterminent alors l'utilité de la photographie en tant que dispositif visuel.

Pourtant, il serait erroné de notre part de remplacer purement et simplement l'hypothèse traditionnelle de la propagation sans difficulté de la photographie par une histoire totalement révisionniste de son étonnante faillite. L'objectif a été ici d'abandonner une narration qui serait celle de l'évidence même (en décrivant l'adoption de la technique) ou de la surprise (en réaction à son absence), et d'accorder plutôt une attention plus soutenue aux circonstances particulières, locales et souvent imprévisibles dans lesquelles la photographie se trouva être digne d'intérêt, ou ignorée, ou encore ponctuellement utilisée. Même si la technique était plus couramment employée en France qu'en Grande-Bretagne, les Français aussi transformaient les photographies en gravures et continuaient à s'appuyer sur d'autres supports tels que les dessins. L'épisode des objets perdus dans le Tigre a en effet montré que le dessin et photographie bénéficiaient d'une la valeur probante à peu près égale, du moins durant ces premières années où se sont rencontrées l'archéologie et la photographie.

\section{L'auteur}

Après avoir suivi un postdoc à l'université de Harvard, au Max Planck Institut pour l'Histoire de la Science et au Kunsthistorisches Institut de Florence, Mirjam Brusius est docteur en histoire de l'art de la Humboldt-Universität zu Berlin, docteur en histoire et philosophie des sciences de l'université de Cambridge. Elle est actuellement « Mellon Fellow » de l'université d'Oxford.

Ses centres de recherches portent sur l'histoire de la photographie, les musées, les collections, les voyages scientifiques en Europe et entre l'Europe et le Moyen Orient. Ses publications récentes et à venir concernent les archives de l'inventeur anglais, homme de science et antiquaire W. H. F. Talbot. Elle écrit également sur les premières manifestations de la photographie en Iran et au Moyen Orient. Elle travaille actuellement à la rédaction de son deuxième livre: The Canon under Threat. Ce projet a pour objectif d'explorer les trouvailles archéologiques de Mésopotamie et les difficultés rencontrées lors de leur transport vers les musées de Paris, Londres et Berlin. 